\title{
Pronósticos demanda de energía y potencia a Corto plazo. Caso de estudio: unidad de Control DE PRonóstico noroeste (Área Antioquia-Chocó).
}

\author{
HÉctor Tabares Ospina ${ }^{1}$ \\ JesÚs HeRnÁNDEZ RIVEROS ${ }^{2}$
}

\section{Resumen.}

El objetivo de este artículo es hacer un estudio del estado del arte referido a la Gestión de la Demanda de Energía y Potencia, caso particular, área operativa Antioquia - Choco, el marco regulatorio y las demás labores anexas como la elaboración semanal del pronóstico de demanda, sus estadísticas e indicadores y la gestión necesaria para asegurar la calidad. Finalmente se presentan propuestas de investigación que se pueden derivar sobre el asunto estudiado.

\section{Palabras claves:}

Pronostico de demanda de energía y potencia, UCP (Unidad de Control de Pronóstico).

\section{Abstract.}

The objective of this paper is to present a study about the state of the art referred to the Short Term Load Forecasting, specific case, Antioquia-Chocó Operative Area, regulatory and other annexed such as weekly preparation of demand prediction, its statistics and indicators and necessary administration to assure quality.

1 M.Sc. Instituto Tecnológico Metropolitano. Docente Ocasional e Integrante Grupo de Investigación GIT, hectortabares@itm.edu.co

2 Ph.D. Universidad Nacional de Colombia. Docente TC. jahernan@unalmed.edu.co 
At the end of this paper, an investigation proposal which can result from this topic is presented.

\section{Keywords:}

Short term load forecasting. 


\section{INTRODUCCIÓN.}

Este trabajo es el inicio de una propuesta de investigación en la línea ENERGÍA Y DESARROLLO DE NUEVAS TECNOLOGÍAS, del programa doctorado en SISTEMAS ENERGÉTICOS, de la Universidad Nacional de Colombia, Sede Medellín. En particular, se están estudiando las series de tiempo de demanda de energía eléctrica, las cuales siguen en la mayor parte de los casos complejas dinámicas no-lineales de carácter caótico.

El objetivo fundamental de este artículo consiste en realizar un estudio sobre los diferentes métodos convencionales utilizados por las empresas del sector eléctrico colombiano como instrumento de modelación y predicción no paramétrica para realizar los pronósticos de la demanda de energía y potencia a corto plazo, en el horizonte de una semana con resolución diaria y en el horizonte de 24 horas con resolución horaria.

El articulo está organizado de la siguiente forma: En la Sección 2 se presenta una revisión del estado del arte en materia de pronósticos de energía y potencia eléctrica, se identifican las variables que inciden en su demanda y se estudia el método empleado por las empresas del sector eléctrico colombiano para calcularla. Seguidamente se presenta un resumen del Código de Operación del SIN (Sistema Interconectado Nacional). En la última sección se muestran las conclusiones y se presentan trabajos futuros.

\section{Materiales y métodos}

\subsection{Proceso para elaborar el pronóstico de demanda.}

\subsubsection{Revisión estado del arte.}

Los modelos de series de tiempo estocásticas y de regresión lineal son ampliamente utilizados en el sector eléctrico, (Shoults et al., 1996); las limitaciones de estos modelos motivan el desarrollo y aplicación de nuevas metodologías para la previsión del consumo de energía eléctrica, que representen, de manera más exacta, las 
curvas de demanda. Dentro de este contexto, en las dos últimas décadas se han introducido nuevos modelos de previsión que manejan de manera eficiente la aleatoriedad de los consumos y presentan una fácil adaptabilidad a nuevos datos, sin la necesidad de incurrir en laboriosas formulaciones matemáticas. Entre estos modelos se incluyen las técnicas de las Redes Neuronales Artificiales (RNA) (Lee et al., 1992; Alireza et al., 1995; Khotanzad et al., 1995; Bakirtzis et al., 1996; Piras et al., 1996; Sforma et al., 1996; Caciotta et al., 1997), los Sistemas Fuzzy (SF), (Miranda \& Saraiva, 1992; Matos et al., 2008) y los sistemas Neuro-Fuzzy (NF) (Kim, 1995; Lotfalian et al., 1998; Kim et al., 2000; Alireza et al., 2002; Ling et al., 2003; Liao et al., 2006).

Estas técnicas han tenido una gran aceptación en el desarrollo de modelos para previsión de series de tiempo del sector eléctrico, debido a que combinan la capacidad de aprendizaje y adaptación de las RNA y el poder de interpretación lingüística de los SF.

\subsubsection{Oferta y demanda.}

Como se explica en (Lozano et al., 2007), uno de los principales impactos de la realización de pronósticos de demanda se presenta sobre la definición del precio de la electricidad en la bolsa de energía, el cual sirve de referencia para las demás transacciones y contratos que se realizan y es clave en la libre competencia del mercado desregulado de energía. Se hace de acuerdo con las reglas definidas en la resolución CREG 024-95 (CREG, 1995a), la cual establece que el ASIC (Administrador de Intercambios Comerciales) definirá para cada hora del día, la lista de los generadores que saldrán despachados, según meritos alcanzados. La lista se elabora con base en la demanda pronosticada para cada hora del día y para cada región del país. Se presentan dos casos cuando ocurren desviaciones en la demanda pronosticada:

Primer caso, si el pronóstico es menor a la demanda real, el CND realiza un redespacho en donde debe usar en la mayoría de los casos plantas y unidades de generación más costosos para atender la demanda. Dada esta situación, los demandantes tendrían la 
posibilidad de esperar a que se atendiera su petición, formando colas de espera. Y como se sabe, la falta de electricidad generada conlleva un retraso en el suministro eléctrico que puede tener graves consecuencias económicas y sociales. $\mathrm{O}$ alternativamente podrían consumir algún sustitutivo cercano al bien demandado. Sin embargo, en el mercado eléctrico no existe la posibilidad de recurrir a sustitutos cercanos, al menos en el corto plazo.

Segundo caso, si el pronóstico fue mayor a la demanda real, se incurre en sobrecostos de operación que involucran gastos por el encendido de unidades térmicas que no se requieren o por haber considerando generaciones de seguridad innecesarios. En este caso, se producen perdidas asociadas a una asignación ineficiente de las fuentes primerias, constituyen recursos escasos y limitados. Por lo tanto, al ser la electricidad un bien no acumulable, el exceso de oferta, es decir la electricidad producida y no consumida inmediatamente, se pierde, produciendo inestabilidad en la red.

\subsubsection{Variables que influyen en la demanda.}

Una serie de investigaciones realizadas sobre la demanda eléctrica y sus determinantes (Murto, 1998) en diferentes países han permitido identificar un conjunto de variables básicas que explica la evolución de la demanda eléctrica a lo largo del tiempo, siendo este conjunto común para la mayoría de los países desarrollados. A continuación se comentan detalladamente las variables seleccionadas como posibles factores explicativos de la curva de carga.

Calendario. Existen diferentes efectos relacionados con el calendario que incide sobre la curva de demanda eléctrica:

- Hora del día. Resulta evidente que la demanda eléctrica realizada a las tres de la madrugada no es igual a la realizada a las 3 de la tarde. En un día convencional, el grueso de la demanda eléctrica se acumula en los periodos comprendidos entre las 10 hasta las 15 horas y entre las 19 hasta 21 horas, algo lógico si se tiene en cuenta que dichos periodos corresponden con la jornada laboral estándar. 
- Día de la semana. Repitiendo el mismo planteamiento, se puede intuir que la demanda de electricidad a la misma hora de días diferentes será también diferente. Así, la demanda llevada a cabo un miércoles a las once de la mañana probablemente no coincida con la realizada un domingo a esa misma hora.

- Fin de semana y fiestas. También se puede observa la presencia de un factor que incide sobre la demanda eléctrica y que está asociada a los fines de semana y a los días no laborales. En estos días se produce una reducción generalizada en el consumo eléctrico.

- Mes del año. En función del mes en el que se encuentre, la demanda diaria media de energía varía significativamente. Esto se debe principalmente al efecto de las estaciones. En invierno, desde mayo 1 hasta noviembre 30, aumenta el uso de sistemas calefactores, mientras que en verano (entre diciembre 1 y abril 30), se experimenta un considerable aumento en el consumo como resultado del uso de sistemas de aire acondicionado.

Los pronósticos realizados por la UCP NOROESTE tienen en cuenta el tipo de día a analizar. Explica Lozano (2007) que en la metodología empleada, se clasifican los días en lunes normal, lunes festivo, martes, miércoles, jueves, viernes, sábado y domingo, para cada periodo del día. También se realiza un manejo especial para los días exclusivos o únicos del año. En el análisis del comportamiento de la demanda se identifican varios días especiales que solo se presentan una sola vez al año, los cuales se pronostican mediante reglas diferentes. Los días con estas características son:

1. Lunes, martes y miércoles de semana santa que presentan una leve disminución en la demanda total del día con respecto a los anteriores del mismo día de la semana, debido principalmente a la disminución del consumo.

2. Viernes y sábado de semana santa, 24, 25, 26 y 31 de diciembre, 1 y 2 de enero presentan una curva de carga diferente a los demás días del año. 
3. Días de vacaciones de fin e inicio de año que depende principalmente de la actividad escolar e industrial, se presentan entre el 20 de diciembre y el 15 de enero La forma de la curva de carga es similar a las anteriores de los mismos días de la semana, pero con una disminución más representativa en la demanda horaria.

4. Día 22 de Abril, El concejo de la ciudad de Medellín aprobó el acuerdo de obligatorio cumplimiento El Día Sin Carro, desde las 6:00 a.m. hasta las 17:00 p.m, con el propósito de crear una conciencia ambiental a favor de disminuir los contaminantes que están contribuyendo al aceleramiento del calentamiento global. Con el propósito de facilitar el desplazamiento de los ciudadanos, el Metro aumento su frecuencia y opero durante toda la jornada con trenes de 6 coches.

Condiciones meteorológicas. Aquí se engloban variables como la velocidad del viento, la nubosidad, la pluviosidad, la temperatura, etc. Sin embargo, no todos los factores climáticos afectan a la demanda eléctrica. Algunos de ellos son típicamente aleatorios y otros aparecen interrelacionados. Por ejemplo, la temperatura viene explicada parcialmente por la nubosidad, la pluviometría, la humedad relativa, etc.

Entre todos los factores anteriormente mencionados, la temperatura es el más relevante, dado que influye de forma directa sobre múltiples fuentes de consumo eléctrico como sistemas calefactores, aire acondicionado, refrigeradores, etc. Esta relación entre la demanda eléctrica y la temperatura es de carácter no lineal.

La temperatura, dado que la inmensa mayoría de los trabajos consultados señalan esta variable como principal determinante meteorológica de la demanda eléctrica, la UCP NOROESTE no la tiene en cuenta para realizar sus pronósticos debido a que por factores regulatorios previsto principalmente en el acuerdo 312 del CNO (CNO, 2004), el pronóstico de energía y potencia horaria se debe enviar semanalmente al CND por los operadores de red de Colombia. En los pronósticos realizado por la UCP NOROESTE, no se tienen en cuenta los datos meteorológicos. Como comenta 
Lozano, la consideración de esta variable es impráctica, ya que implicaría depender de su pronóstico con un horizonte temporal igual al que se requiere para la predicción de la demanda: de 7 días, y las entidades ambientales como CORANTIOQUIA o el IDEAM que hacen el pronóstico de estas variables tienen un margen de error del 5\% en sus predicciones para el día siguiente. Esto indica que extenderlo a un periodo más largo incrementaría las posibilidades de error, propagándolo al pronóstico de energía y potencia horarias.

Información económica. Dada la extensa penetración de la electricidad en la actividad económica del país, existen multitud de variables económicas que influyen en la demanda. La mayoría de ellas covarían entre sí en mayor o menor medida. Introducir varias por separado en un modelo explicativo da lugar a problemas de multilinealidad que haría imposible lograr unos resultados aceptables.

Si bien todas estas variables pueden incidir significativamente en la demanda eléctrica, la UCP NOROESTE opto por no incluirlas en su modelo para realizar pronósticos, por cuanto la mayor parte de ellas afectan a la demanda, pero sus efectos son apreciables en el mediano y largo plazo, siendo irrelevante en el corto plazo, horizonte sobre el que se realizan los pronósticos de demanda de energía.

Factores no predecibles. Se debe considerar la existencia de una serie de factores, que siendo puramente aleatorios, pueden afectar sensiblemente a la demanda de energía eléctrica. Entre dichos factores se destacan el cierre de instalaciones industriales intensivas en electricidad, desastres naturales, climatología adversa, presentación de averías o atentados terroristas que afectan a la infraestructura eléctrica. Por su carácter impredecible, la UCP NOROESTE no tiene en cuenta estos factores dentro de los análisis de pronósticos.

Información ausente. Algunos científicos han llamado "la información ausente", la incapacidad para hacer una descripción completa de un fenómeno. Esta información puede ser de gran 
importancia, pero está ausente. En este sentido, existen factores desconocidos cuyo efecto sobre la demanda eléctrica puede ser considerado como un "ruido blanco". La UCP NOROESTE, para efectos prácticos, filtra esta información dentro del análisis de pronósticos.

Como conclusión final, aunque se sabe que el consumo de energía eléctrica es función funciona de las variables independientes: el calendario, las condiciones meteorológicas, información económica, factores no predecibles e información ausente, la UCP NOROESTE para efectos prácticos, las filtra, a pesar de que la mayoría de ellas covarían entre sí en mayor o menor medida, quedando el calendario como única variable que alimenta el modelo de pronóstico utilizado.

\subsection{Código de operación del sin.}

Un detallado estudio sobre el código de redes está más allá del ámbito de esta unidad. En (CREG, 1995b) se ofrece una completa información. Un recuento de los principales antecedentes relacionados con este tema, se presentan a continuación:

- Mediante la ley 134 de 1994 (Art 38) y el Acuerdo $\mathrm{N}^{\circ} 20$ del 27 de mayo de 1999 (CNO, 1999) del Consejo Nacional de Operación (CNO), estableció el procedimiento para el cálculo y envío de la demanda de las áreas operativas del SIN al Centro Nacional de Despacho (CND) y adicionalmente se establecieron las responsabilidades de los Centros Regionales de Despacho (CRD) relacionadas con la supervisión y control de la operación de las redes, subestaciones y centrales de generación localizadas en una misma región.

- La resolución de la Comisión de Regulación de Energía y Gas (CREG) 025 de 1995 (CREG, 1995b), estableció que el manejo de la demanda se debe realizar por Áreas Operativas y debe obedecer al siguiente criterio "Un Área operativa comprende un conjunto de subestaciones, recursos de generación y demanda que presentan alguna restricción eléctrica que limitan los intercambios con el resto del sistema”. 
- La gestión de la demanda, tal como se establece en el marco de la Ley Eléctrica, resolución CREG 080 de 1999 (CREG, 1999), es realizada por los Operadores de Red (OR'S). Como consecuencia de lo anterior y como lo estipulan los Acuerdos $\mathrm{N}^{\circ} 126$ de 2001 (CREG, 2001) y 349 de 2006 (CNO, 2006a) del CNO, los agentes del mercado acordaron manejar la gestión de la demanda mediante la forma de Unidades de Control de Pronóstico (UCP). Una UCP se define, en lo fundamental, como una porción de demanda que es alimentada por uno o varios Operadores de Red. Cada UCP representa una porción de demanda nacional y tiene un agente responsable de: reportar la demanda de potencia, la demanda no atendida y los factores de distribución de energía activa y de potencia. Una de estas UCP es la llamada NOROESTE, ubicada en el Centro Regional de Control (CRC) de las Empresas Publica de Medellín (EPM), que agrupa la demanda de los departamentos de Antioquia y Chocó.

- La razón primordial para gestionar la demanda por UCP es para tipificar el comportamiento semanal y mensual del pronóstico, con el ánimo de tener elementos de juicio en el caso que la CREG decida reglamentar las desviaciones al pronóstico de la demanda. En este mismo sentido la CREG, por medio de la resolución 095 de 2003 (CREG, 2003), solicito un estudio técnico a cada UCP que incluya criterios que permitan, entre otros, ajustar y optimizar el proceso de pronóstico de la demanda.

\subsubsection{Características de la información de demanda}

- Nomenclatura: Se elimina el concepto de hora al referirse en todo lo relacionado con la demanda, tanto energía como de potencia y se establece el concepto de periodo.

- Definición de punta: Dado que del análisis de curvas de carga no se observa que en las horas de la mañana exista una variación significativa que implique tratamiento especial de 
ningún periodo, solo se adopta como punta la de la tarde, que corresponde a los periodos 19, 20 y 21 .

- Programa despacho económico horario. Es el programa de generación de las unidades SIN en cada una de las horas del día, producido por el despacho económico.

- Información a utilizar en el despacho económico: Para efectos del despacho económico, se adaptara la información de demanda real, para alimentar los modelos de pronóstico a un formato horario con las siguientes características: En los periodos de punta, el dato a emplear debe corresponder a la potencia pronosticada para cada periodo. Esto con el objeto de garantizar la seguridad del sistema en los periodos de máxima carga o similares. En los periodos fuera de punta, el dato a emplear debe corresponder a la energía pronosticada para cada periodo.

- Representación de la información para el despacho económico: La curva de carga de la UCP NOROESTE está dada por el diseño presentado en la figura 1, donde para los periodos fuera de punta se registran lecturas de energía MWh, y en los periodos de punta se tienen lecturas de potencia con intervalos de 15 minutos.

- Información histórica de energía: De acuerdo con los lineamientos del CNO, es deseable que los agentes del sistema manejen curvas de carga y de pronóstico con 96 valores diarios, uno cada 15 minutos. Con ello se pretende incrementar la calidad de la información como tal.

- Información histórica de potencia: Los agentes del sistema también deben manejar las medidas de potencia en sub-períodos de 15 minutos para los periodos de punta. Cuando se requiera relacionar la demanda del sub-período con el periodo horario, por ejemplo para el despacho económico, se acordó tomar el máximo de las cuatro lecturas de los sub-período correspondientes y a partir de estos, obtener el valor representativo para el periodo. 


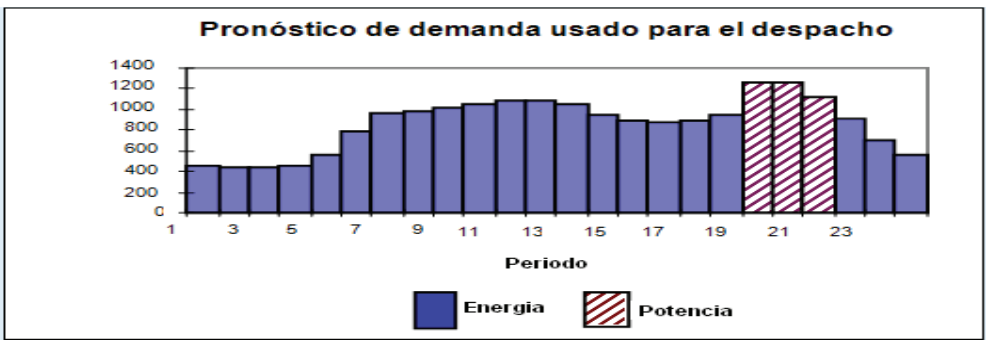

Figura 1. Curva de carga UCP NOROESTE, usado para El DESPacho.

- Horizonte de Planeamiento Operativo. Es el periodo de tiempo cubierto por cada una de las etapas del planeamiento operativo denominadas Largo, Mediano Plazo, Corto Plazo y muy corto Plazo. El horizonte del Largo Plazo es de cinco años. El Mediano Plazo de cinco semanas, el Despacho Económico de 24 horas y el Muy Corto Plazo desde la hora actual hasta el final del día.

- La planeación de corto plazo. Comprende la recepción de las ofertas diarias que presentan los generadores en la Bolsa de Energía, donde se asignan hora a hora las plantas que suministrarán la energía al día siguiente. El CND, realiza esta selección con criterios de seguridad y economía para garantizarle a los usuarios el servicio con estándares de calidad, confiabilidad y eficiencia.

- Gestión de la Demanda. Tiene como finalidad analizar y caracterizar la demanda de energía del sistema, construir pronósticos de energía y potencia para diferentes resoluciones temporales y espaciales, distribuir y administrar posibles casos de programación de racionamiento.

- Periodos estacionales. El planeamiento operativo caracteriza dos estaciones: verano, comprendido entre diciembre 1 y abril 30 . Invierno, entre mayo 1 y noviembre 30.

- Declaración de datos hidrológicos. Con el propósito de evaluar las reservas energéticas, las empresas de generación, las propietarias de plantas hidráulicas deben informar diariamente al CND antes de las 07:00 horas de cada día los siguientes datos: nivel 
del embalse, agua turbinada, agua vertida, agua descargada, el promedio de aportes, relación de mantenimiento.

- Perdidas. Las pérdidas horarias del Sistema de Transmisión Nacional (STN) se calculan como la diferencia entre la energía inyectada y extraída del STN. Las pérdidas así calculadas para cada hora del día $(k)$ son consideradas dentro del despacho horario para la hora correspondiente del día $(k+2)$.

- Balance de la generación, la demanda y las pérdidas del Sistema Interconectado y de los intercambios internacionales. Determinación del punto de equilibrio entre la oferta y la demanda en el mercado de energía, teniendo en cuenta las pérdidas del sistema, con el fin de establecer la magnitud de las compensaciones de agentes comercializadores y generadores.

\subsubsection{Pronóstico de la demanda}

Según lo estipulado en la resolución CREG 025 de 1995 (CREG, 1995b), Código de Operación del Código de Redes, numeral 3.1, Despacho económico horario, Información básica de demanda, "La predicción horaria para el Despacho Económico se efectúa por aéreas operativas y para cada una de las 24 horas de cada día de la semana. Esta predicción de demanda de potencia la efectúa en CND y la envía a los agentes del sistema semanalmente el día miércoles y recibe comentarios o modificaciones hasta el día viernes a las 13:00 horas".

Por lo tanto, en la actualidad cada agente del sistema envía al CND el pronóstico antes de las 9:00 de cada día viernes y cubre una ventana de tiempo de la semana siguiente. El pronóstico consta de:

- Pronóstico horario de la demanda de energía (MWh), para cada una de las 24 horas de cada día.

- Pronóstico de la demanda esperada de potencia (MW), para las tres horas de punta (periodos 19, 20 y 21) de cada día.

Una vez elaborada la demanda, el CND realiza el pronóstico oficial utilizando para tal fin los promedios móviles de la serie histórica con los datos horarios de energía desde el año 2005 y para 
esto se ajustan los modelos propuestos tomando como horizonte alrededor de las 15 semanas anteriores y proyectando la semana siguiente que es la pronosticada. A los datos resultantes para un día particular se les puede aplicar suavización exponencial.

El CNO por medio del Comité de Distribución y con el objeto de mejorar el Pronóstico de Demanda Diario utilizado para el despacho económico, consideró el antecedente de que todas las investigaciones efectuadas muestran que reducir la restricción en el tiempo de anticipación del Pronostico, mejora significativamente la calidad del mismo. CNO, Acuerdos 366 de 2006 (CNO, 2006b) y 370 de 2006 (CNO, 2006c).

\subsubsection{Despacho económico horario}

Es el proceso mediante el cual se obtiene para un periodo de 24 horas el programa horario de generación de los recursos del SIN despachados centralmente, como según lo indican las resoluciones CREG 053 de 1994 (CREG, 1994a), 055 de 1994, Art. 12-15 (CREG, 1994b) y 070 de 1995, Art. 1 (CREG, 1995c).

\subsubsection{Cálculo del despacho económico}

El CND actúa como regulador, coordinando en todo momento la oferta y la demanda, asumiendo una serie de responsabilidades vitales:

- La sustitución de energía que un grupo no puede producir.

- El suministro instantáneo de la energía que los consumidores necesitan en cada momento. El esquema de funcionamiento seguido por el CND es el siguiente: En primer lugar averigua el valor que toma la demanda eléctrica en cada momento, tratando de llegar a la denominada curva de carga (Valor que toma la demanda eléctrica en cada momento, se correspondería con la demanda instantánea de energía eléctrica). Una vez conocido este dato, el operador se dirige a los centros de generación, indicando la carga eléctrica que deben de introducir en la red en cada momento para garantizar la cobertura de la demanda, 
y al mismo tiempo, minimizar la sobreproducción y sus costos de eficiencia.

Para cada una de las horas del día el CND establece el programa horario de generación de tal forma que se cubra la demanda esperada con los recursos de generación disponibles más económicos ofertados por las empresas, cumpliendo las restricciones técnicas y eléctricas de las unidades generadoras. Resolución CREG112-98-Art: 17 (CREG, 1998).

\section{Resultados y discusión.}

\subsection{Demanda de energía eléctrica del área antioquia-choco en un periodo de 24 horas.}

En la elaboración del pronóstico de demanda de energía y potencia de la UCP NOROESTE, se tiene en cuenta la serie histórica de demanda horaria real (NOROESTE - OFI - Ene 2005-2007), obtenido a partir de los contadores de importación y exportación de la UCP y validada por el CND. En forma general, las curvas de demanda de una semana normal es como se ilustra en la figura 2. Muestra como el consumo de electricidad tiene un fuerte patrón estacional diario que se puede apreciar en todos los días laborales debido a que poseen un perfil de demanda muy similar. Con respecto a los fines de semana se observa cómo, si bien se repite el mismo patrón horario de consumo detectado en los días laborales, en general el consumo medio es sensiblemente menor.

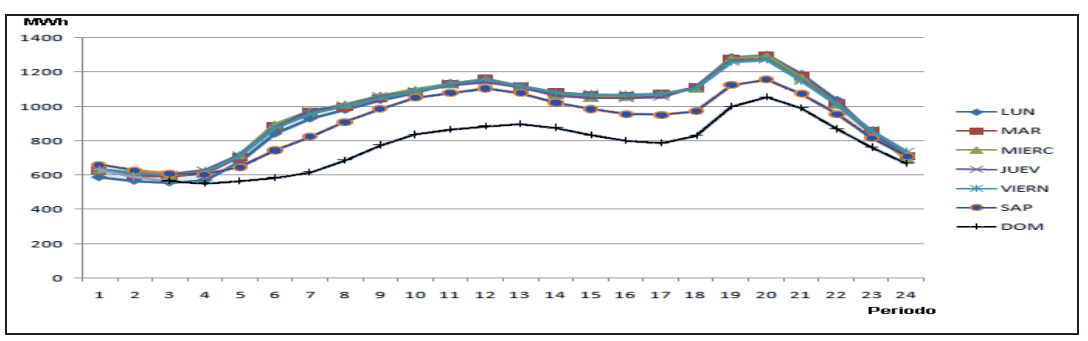

Figura 2. Demanda semanal UCP Noroeste. 
Como se comenta en (Nahmias, 2007), las anteriores series de tiempo son estacionales, porque tiene un patrón de repetición regular durante el mismo periodo de tiempo. Su comportamiento periódico, es reflejado en parámetros como la media, desviación estándar, asimetría, y asimetría de autocorrelación. Y dada la importancia de su previsión, gran variedad de modelos han sido propuestos durante las últimas décadas, de los cuales los más usados son: Regresión Lineal, Medias Móviles, Curvas Típicas Normalizadas, Modelos ARIMA, Suavización Exponencial y Redes Neuronales Artificiales.

Estas técnicas se basan en métodos estadísticos y extrapolan el comportamiento de los consumos en el pasado, mientras consideran el efecto de otros factores como el clima y la correlación especial entre consumos de diferentes días; sin embargo, estos modelos emplean un gran número de relaciones complejas y no lineales entre las series de consumos; se requiere además una gran cantidad de tiempo y esfuerzo computacional, y pueden resultar soluciones numéricamente inestables.

Por consiguiente, el método utilizado por la UCP NOROESTE para hacer pronósticos está basado en técnicas estadísticas convencionales básicas, con las que para obtener unos resultados aceptables se requiere posteriormente la actuación de un experto, cuyo papel es ajustar continuamente los múltiples parámetros del programa de pronóstico, en especial, los causados por cambios en la estructura del mercado.

La información que envía el CND a la UCP NOROESTE para hacer pronósticos, en su estado original tiene el siguiente formato.

TABLA 1. INFORMACIÓN PRONÓSTICO DEMANDA DE ENERGÍA ELÉCTRICA UCP NOROESTE.

\begin{tabular}{|l|l|l|l|l|l|l|l|l|l|}
\hline \multicolumn{1}{|c|}{ FORMATO PRONÓSTICO DEMANDA DE ENERGÍA ELÉCTRICA } \\
\hline Reg & FECHA & P1 & P2 & $\ldots$ & P24 & TOTAL & PO19 & PO20 & PO21 \\
\hline 1 & $01 / 01 / 2005$ & 687 & 607 & & 493 & 14.955 & 924 & 979 & 900 \\
\hline 2 & $02 / 01 / 2005$ & 512 & 456 & & 583 & 17.620 & 1.025 & 1.090 & 1.020 \\
\hline & & & & & & & & & \\
\hline 1127 & $31 / 01 / 2008$ & 642 & 611 & & 730 & 23.039 & 1.338 & 1.351 & 1.236 \\
\hline
\end{tabular}


Los resultados de las predicciones se presentan en la Tabla 1. Se interpreta P1, como el periodo desde 0:00 p.m. hasta las 0:59 a.m. Por lo tanto el día se desagrega en 24 periodos horarios del 1 al 24. Se registra la potencia activa (MWh) en cada periodo, el total registrado durante el día y los máximos consumos de energía, registrados en la punta de la serie (periodos 19, 20 y 21).

La UCP NOROESTE registra en otra tabla, pero con el mismo formato de la Tabla 1, los consumos reales de potencia activa registrados en los contadores en cada uno de los periodos del día, el total registrado durante el día y los máximos consumos de energía registrados en los periodos 19, 20 y 21 (punta de la serie).

Seguidamente la UCP NOROESTE calcula la diferencia entre el pronóstico para el periodo y la demanda real para el mismo periodo. Se entiende que toda diferencia negativa corresponde a un exceso de oferta, lo que se traduce en una asignación ineficiente de los recursos. Así mismo, toda diferencia positiva significa que hubo un exceso de demanda, lo que pudo ocasionar un retraso en el suministro.

Finalmente se evalúa el desempeño del pronóstico calculando los errores en las predicciones de cada periodo del día. La medida más común de exactitud de pronósticos durante los 24 periodos es el error cuadrático medio (ECM).

Se pretende que los errores aceptables en las desviaciones no superen el $6 \%$ por exceso de demanda o el $-6 \%$ por exceso de oferta. Estudiando la tabla 2, en el periodo comprendido entre los años 2005 a 2008, se observa que el universo de errores cometidos en los pronósticos, 23097 casos, se encuentran en el intervalo [-6, 6]. Optimizando los métodos de pronósticos se pretende disminuir la cantidad de errores que se encuentran por fuera del intervalo antes indicado, 3918 casos, sabiendo que la demanda tiene muchas posibilidades de ser aleatoria bajo muchas circunstancias y entre más alejado sea el pronóstico, menos exacta será la predicción. 
Tabla 2. Histograma PRON_OFI UnOROESte (01/01/2005 - 31/01/2008)

\begin{tabular}{|c|c|c|c|c|c|c|c|c|c|c|c|c|c|c|c|c|c|c|c|c|c|c|c|c|c|c|c|}
\hline \multicolumn{28}{|c|}{ HISTOGRAMA } \\
\hline 1 & MUESTRA TOTAL & P1 & P2 & P3 P & P4 F & P5 & P6 P & P7 P & P8 P & Pg & P10 P & P11 P & P12 P & & P14 P & P15 F & P16 P & & P18 F & P19 F & & P21 P & P22 & P23 F & P24 & Suma & \\
\hline 2 & Menores que $-20 \%$ & 0 & 0 & 0 & 0 & 1 & 1 & 6 & 3 & 2 & 2 & 1 & 1 & 1 & 0 & 0 & 0 & 0 & 0 & 0 & 0 & 0 & 0 & 0 & 0 & 18 & 0 \\
\hline 3 & $-20 \%--18 \%$ & 1 & 0 & 0 & 0 & 0 & 3 & 0 & 0 & 1 & 1 & 1 & 1 & 0 & 0 & 0 & 0 & 0 & 1 & 0 & 0 & 0 & 1 & 1 & 0 & 11 & 0 \\
\hline 4 & $-18 \%--16 \%$ & 2 & 3 & 2 & 1 & 0 & 1 & 1 & 0 & 0 & 0 & 0 & 0 & 2 & 1 & 0 & 0 & 1 & 0 & 1 & 1 & 0 & 2 & 6 & 6 & 30 & 0 \\
\hline 5 & $-16 \%--14 \%$ & 6 & 2 & 2 & 3 & 0 & 3 & 4 & 5 & 4 & 3 & 1 & 1 & 2 & 0 & 0 & 0 & 0 & 1 & 0 & 1 & 2 & 8 & 16 & 12 & 76 & 0 \\
\hline 6 & $-14 \%--12 \%$ & 14 & 6 & & 4 & 5 & 5 & 8 & 8 & 3 & 1 & 1 & 3 & 1 & 2 & 2 & 2 & 4 & 4 & 0 & 0 & 7 & 15 & 28 & 20 & 147 & 1 \\
\hline 7 & $-12 \%--10 \%$ & 26 & 27 & 21 & 7 & 9 & 25 & 13 & 8 & 8 & 7 & 6 & 1 & 3 & 12 & 5 & 3 & 4 & 8 & 2 & 6 & 14 & 48 & 43 & 53 & 359 & 1 \\
\hline 8 & $-10 \%--8 \%$ & 56 & 37 & 51 & 36 & 13 & 25 & 47 & 24 & 15 & 16 & 21 & 19 & 38 & 43 & 18 & 15 & 13 & 18 & 8 & 19 & 70 & 99 & 95 & 80 & 876 & 3 \\
\hline 9 & $-8 \%--6 \%$ & 119 & 86 & 74 & 62 & 51 & 61 & 87 & 74 & 56 & 58 & 49 & 87 & 136 & 111 & 64 & 49 & 44 & 68 & 27 & 91 & 153 & 170 & 170 & 145 & 2092 & 8 \\
\hline 10 & $-6 \%--4 \%$ & 183 & 154 & 142 & 122 & 110 & 117 & 169 & 1901 & 148 & 183 & $\overline{2112}$ & 250 & 272 & 265 & 169 & 144 & 123 & 141 & 81 & 288 & 294 & 232 & 200 & 180 & 4368 & $\overline{16}$ \\
\hline 11 & $-4 \%--2 \%$ & 242 & 259 & 2402 & 200 & 182 & 192 & 2763 & 3113 & 312 & 370 & 3763 & 395 & 340 & 313 & 328 & 287 & 288 & 217 & 216 & 403 & 315 & 248 & 218 & 212 & 6740 & 25 \\
\hline 12 & $-2 \%-2 \%$ & 331 & 409 & 424 & 491 & 462 & 454 & 435 & 4545 & 539 & 471 & 4353 & 345 & 298 & 345 & 492 & 542 & 521 & 413 & 617 & 296 & 242 & 243 & 256 & 282 & 9797 & 36 \\
\hline 13 & $2 \%-4 \%$ & 87 & 87 & 115 & 133 & 187 & 147 & 54 & 37 & 31 & 11 & 18 & 15 & 26 & 24 & 32 & 63 & 84 & 136 & 116 & 16 & 18 & 39 & 56 & 68 & 1600 & 6 \\
\hline 14 & $4 \%-6 \%$ & 37 & 38 & 34 & 50 & 76 & 60 & 15 & 10 & 7 & 2 & 5 & 6 & 2 & 7 & 12 & 16 & 30 & 62 & 42 & 3 & 8 & 11 & 21 & 38 & 592 & 2 \\
\hline 15 & $6 \%-8 \%$ & 11 & 11 & 8 & 10 & 22 & 19 & 5 & 1 & 0 & 1 & 1 & 1 & 3 & 1 & 4 & 4 & 9 & 34 & 12 & 1 & 3 & 8 & 10 & 16 & 195 & $\sqrt{1}$ \\
\hline 16 & $8 \%-10 \%$ & 6 & 4 & 6 & 4 & 5 & 7 & 4 & 1 & 0 & 0 & 0 & 0 & 1 & 1 & 0 & 0 & 4 & 16 & 4 & 0 & 0 & 1 & 4 & 6 & 74 & 0 \\
\hline 17 & $10 \%-12 \%$ & 3 & 1 & 1 & 2 & 2 & 4 & 2 & 0 & 0 & 0 & 0 & 1 & 0 & 0 & 0 & 1 & 1 & 3 & 0 & 0 & 0 & 0 & 2 & 5 & 28 & 0 \\
\hline 18 & $12 \%-14 \%$ & 0 & 1 & 2 & 1 & 1 & 1 & 0 & 0 & 0 & 0 & 0 & 0 & 1 & 0 & 0 & 0 & 0 & 3 & 0 & 0 & 0 & 1 & 0 & 2 & 13 & 0 \\
\hline 19 & $14 \%-16 \%$ & 1 & 1 & 0 & 0 & 0 & 1 & 0 & 0 & 0 & 0 & 0 & 0 & 0 & 1 & 0 & 0 & 0 & 0 & 0 & 0 & 0 & 0 & 0 & 1 & 5 & 0 \\
\hline 20 & $16 \%-18 \%$ & 1 & 0 & 0 & 0 & 0 & 0 & 0 & 0 & 0 & 0 & 0 & 0 & 0 & 0 & 0 & 0 & 0 & 0 & 0 & 1 & 0 & 0 & 0 & 0 & 2 & 0 \\
\hline 21 & $18 \%-20 \%$ & 0 & 0 & 0 & 0 & 0 & 0 & 0 & 0 & 0 & 0 & 0 & 0 & 0 & 0 & 0 & 0 & 0 & 1 & 0 & 0 & 0 & 0 & 0 & 0 & 1 & 0 \\
\hline 22 & Mayores que $20 \%$ & 0 & 0 & 0 & 0 & 0 & 0 & 0 & 0 & 0 & 0 & 0 & 0 & 0 & 0 & 0 & 0 & 0 & 0 & 0 & 0 & 0 & 0 & 0 & 0 & 0 & 0 \\
\hline
\end{tabular}

Finalmente, se calcula el promedio de los errores cometidos en los pronósticos realizados durante cada mes del periodo analizado, cada día con 24 registros. Se calcula la desviación estándar del universo de registros del mes con respecto al promedio mensual, con el objeto de medir su grado de dispersión. Una desviación estándar grande indica que los errores en las predicciones alcanzados están lejos de la media, y una desviación pequeña indica que los errores están agrupados cerca de la media. Si la media de las medidas está demasiado alejada de la desviación estándar, entonces se considera que el método empleado para hacer los pronósticos no es bueno. Esto es coherente, ya que las mediciones caen fuera del rango de valores en el cual sería razonable esperar que ocurriera si el procedimiento empleado para hacer los pronósticos fuera correcto. Así mismo, la desviación estándar muestra la agrupación del universo de registros de errores en los pronósticos alrededor de un valor central (la media o promedio). 


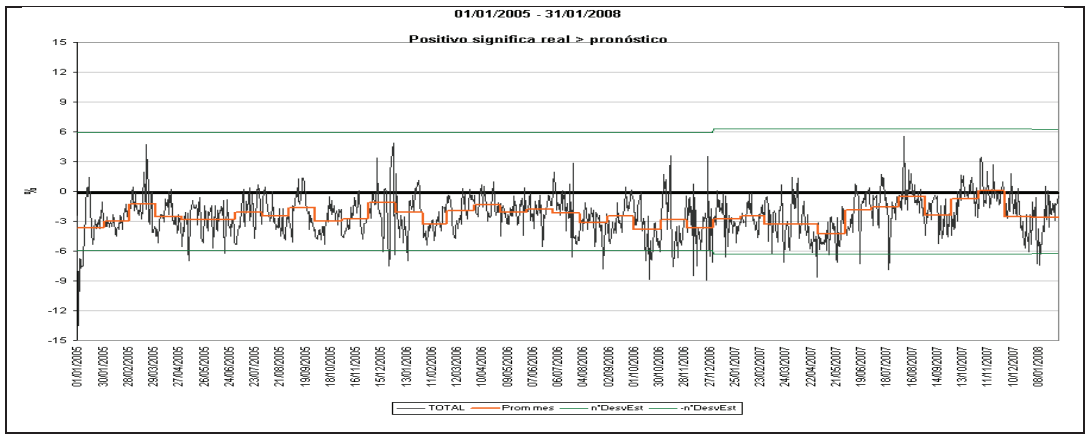

Figura 3. Tendencia consumo energía eléctrica 01/01/05 - 31/01/08. EPM.

La figura 3 presenta el registro de la TENDENCIA CONSUMO DIARIO de la UCP NOROESTE, desde el 01/01/2005 hasta el 01/31/2008, para un total de 1127 días. Se grafica el error total del pronóstico de cada día, el promedio del mes y su desviación estándar. Los registros totales positivos significan que el consumo real fue mayor que el pronosticado.

Este último tipo de serie generada es de tipo caótico, por las siguientes razones: no posee ningún rasgo de estacionalidad, no demuestra tener ningún tipo de tendencia marcado, no tiene puntos fuera del rango y parece ser estable en la media. Su comportamiento caótico se explica porque el pronóstico es función de, al menos, 5 variables linealmente independientes como son: El calendario, las condiciones meteorológicas, factores no predecibles, factores económicos y la información ausente. Por lo tanto resulta interesante estudiar observaciones que se desprenden de su análisis caótico como seria su dimensión fractal, orbitas, atractores y su posible interpretación en el sector eléctrico.

\section{Conclusiones}

La demanda eléctrica es muy variable a lo largo de los años, los meses, los días e incluso las horas. Estas características aportan una incertidumbre al sistema eléctrico que afecta de forma significativa el costo final del suministro. Para reducir este factor 
de coste, el gestor necesita conocer con precisión los mecanismos que hacen variar la demanda.

Con la información aportada por la curva de carga se logran dos objetivos. En primera instancia, prever y adaptarse a la demanda en los distintos plazos (a corto, en la operación del sistema, y a largo en la creación de nuevas centrales y redes para el suministro). En segundo caso, gestionar la demanda, es decir, inducir a los consumidores a modificar sus pautas de consumo eléctrico para lograr una mayor eficiencia conjunta del proceso de suministro y uso.

Por lo tanto, el objetivo fundamental que se pretende con este trabajo es ajustar y optimizar el proceso de pronóstico de la demanda, como según consta en la resolución CREG 095 de 2003 (CREG, 2003). Como lo indican los acuerdos 366 y 370 de 2006 de la CNO (CNO, 2006b; 2006c), todas las investigaciones efectuadas hasta el presente muestran que reducir la restricción en el tiempo de anticipación del pronóstico, mejora significativamente la calidad del mismo.

Con esta propuesta de investigación no se pretende suplantar ninguna de las metodologías existentes, sino más bien abrir el campo de investigación respecto al estándar, en lo que a este tipo de series se refiere. Esto posibilita la elevación de nuevos planteamientos fundamentales para el desarrollo de la teoría de pronósticos en el sector eléctrico, tales como:

- Disminuir las desviaciones horarias en el pronóstico mediante un modelo que contemple su carácter caótico.

- Modelación e implementación de sistemas Neurodifusos para mapear las series históricas de consumo de energía y ajustar de forma optima los múltiples parámetros del programa de pronósticos, en especial, los causados por cambios en la estructura del mercado.

- Clusterización o agrupamiento de pronósticos. La tecnología Neuro-Difusa provee un método poderoso para convertir datos experimentales de pronósticos en reglas difusas. Sin embargo en muchos casos, los datos de entrenamiento deben ser agrupados 
previamente para eliminar redundancias y conflictos en el caso en que el conjunto de datos sean inconsistente.

Para terminar, es necesario comentar que el principal impacto de esta propuesta de investigación es de tipo social, por cuanto en la medida en que no se optimicen los procedimientos de pronósticos de demanda, conduce a sobrecostos que incrementan el precio de la energía para el usuario final y disminuye la capacidad de competir con precios más bajos en el mercado de la energía.

Agradecimientos: Esta sección reconoce la ayuda de los Ingenieros Giovanny Marín Avalos y Nelson Valencia Zapata, Profesionales Centro Regional de Control (CRC), EPM, por su asistencia, sugerencias e ideas para explicar los pronósticos de demanda de energía y potencia. Se agradece a los revisores del artículo las constructivas sugerencias realizadas.

\section{Referencias bibliográficas}

alireza, K. Maratukulam, D. Abaye, A. (1995). An adaptive modular artificial neural network hourly load forecasrter and its implementation at electric utilities. IEEE. Vol 10, No 3, Aug.

Alireza, K. Enwang, Z. Elragal, H. (2002) A Neuro Fuzzy approach to short term load forecasting in a price sensitive environment. IEEE, Vol. 17, No 4 , Nov.

Bakirtzis, G. Petridis, V. Kiartzis, SJ. (1996) A neural network short term load forecasting model for the Greek, power system. IEEE, Vol 11, No 2, May.

Caciotta, M. Lamedica, R. Orsolini, V. (1997) Application of Artificial Neural Networks to Historical Data Analysis for short term electric load forecasting. ETEP, Vol 7, No 1. Jan.

CNO. (1999) Consejo Nacional de Operación, Acuerdo 020 de 1999, Mayo 27, Por el cual se aprueba el procedimiento para la implementación de la demanda en áreas operativas. Bogotá.

CNO (2004) Consejo Nacional de Operación, Acuerdo 312 de 2004, Diciembre 17, Reglamentación sobre los procedimientos de creación, modificación y operación de las Unidades de Control de Pronostico de demanda. Bogotá. 
CNO (2006a) Consejo Nacional de Operación, Acuerdo 349 de 2006, Enero 30, Por el cual se aprueban los procedimientos de creación, operación y modificación de las Unidades de Control de pronósticos de demanda y la oportunidad de reporte de información al CND. Bogotá.

CNO (2006b) Consejo Nacional de Operación, Acuerdo 366 de 2006, Julio 27, Por el cual se acuerda un procedimiento para el suministro de la información de demanda para la elaboración del Despacho económico. Bogotá.

CNO (2006c) Consejo Nacional de Operación, Acuerdo 370 de 2006, Septiembre 28, Por el cual se modifica el artículo 4 del acuerdo 366 sobre el procedimiento para el suministro de la información de demanda diaria para la elaboración del Despacho económico. Bogotá.

CREG (1994a), Comisión de Regulación de Energía y Gas, Resolución 053 de 1994, Artículo 11, Diciembre 28, Por la cual se dictan disposiciones para el funcionamiento del mercado mayorista de energía durante el período de transición hacia un mercado libre y se modifica parcialmente el Acuerdo Reglamentario para el Planeamiento de la Operación del Sistema Interconectado Nacional. Bogotá.

CREG (1994b), Comisión de Regulación de Energía y Gas, Resolución 055 de 1994, Artículos 12-15, Diciembre 28, Por la cual se regula la actividad de generación de energía eléctrica en el sistema interconectado nacional. Bogotá.

CREG (1995c), Comisión de Regulación de Energía y Gas, Resolución 070 de 1995, Artículo 1, Diciembre 21, Por la cual se establecen medidas transitorias para reglamentar el manejo y control de la demanda en situaciones de racionamiento de emergencia, que regirán hasta la expedición oficial del Estatuto de Racionamiento. Bogotá.

CREG (1995a), Comisión de Regulación de Energía y Gas, Resolución 024 de 1995, Julio 10, Por la cual se reglamentan los aspectos comerciales del mercado mayorista de energía en el sistema interconectado nacional, que hacen parte del Reglamento de Operación. Bogotá.

CREG (1995b), Comisión de Regulación de Energía y Gas, Resolución 025 de 1995, Julio 13, Por la cual se establece el Código de Redes, como parte del Reglamento de Operación del Sistema Interconectado Nacional. Bogotá.

CREG (1998), Comisión de Regulación de Energía y Gas, Resolución 112 de 1998, Art. 17, Septiembre 29, Por la cual se reglamentan los aspectos comerciales aplicables a las transacciones internacionales de energía, 
que se realizan en el mercado mayorista de electricidad, como parte integrante del Reglamento de Operación. Bogotá.

CREG (1999), Comisión de Regulación de Energía y Gas, Resolución 080 de 1999, Diciembre 22, Por la cual se reglamentan las funciones de planeación, coordinación supervisión y control entre el Centro Nacional de Despacho (CND) y los agentes del SIN. Bogotá.

CREG (2001), Comisión de Regulación de Energía y Gas, Acuerdo 126 de 2001, Marzo 29, Por el cual se aprueba el documento "Propuesta de evaluación de factores de distribución de energía activa y factores de potencia”. Bogotá.

CREG (2003), Comisión de Regulación de Energía y Gas, Resolución 095 de 2003, Septiembre 25, Por la cual se solicita al Consejo Nacional de Operación y al Centro Nacional de Despacho un estudio técnico sobre el servicio de Regulación Secundaria de Frecuencia -AGC-. Bogotá.

Khotanzad, A. Davis, A. Abaye, MH. Maratukulam A, (1995) An artificial neural network hourly temperature forecaster with applications in load forecasting. IEEE, Vol 11, No 2, May.

Kim, K. (1995) Implementation of Irbid short term load forecasting system using artificial neural network and fuzzy expert systems. IEEE. Vol 10 No 3, Aug.

Kim, K. Youn, HS. Kang, YC. (2000). Short term load forecasting for special days in anomalous load conditions using Neural Networks and Fuzzy Inference Method. IEEE, Vol 15, No 2, May.

Lee, K. Cha, YT. Park, JH. (1992) Short term load forecasting using an artificial neural network. IEEE, Vol 7, No 1, Feb.

Liao, G. Tsao, TP. (2006) Applications of a Fuzzy Neural Network combined with a Chaos genetic algorithm and simulated annealing to short term load forecasting. IEEE Vol 10, N0. 3, June 2006.

Ling, SH. Leung, FHF. Lam, KH. Tam, PKS. (2003) Short Term Electric Load Forecasting Base on Neural Fuzzy. IEEE. Vol. 50, No 6, December.

Lotfalian, M. (1998) Neural network with fuzzy set based classification for short term load forecasting. IEEE, Vol 13, No 4, Nov.

Lozano, C. Valencia, A. Moreno, C. (2007) Modelo de promedios móviles para el pronóstico horario de potencia y energía eléctrica. Revista EL HOMBRE Y LA MAQUINA. UAO. ISSN 0121-0777, No 029, pp. 96-105, Julio. 
Matos, M. Gouveia, E. (2008) The fuzzy power flow revisited. IEEE. Vol 23, No 1, Feb.

Miranda, V. Saraiva, JP. (1992) Fuzzy modeling of power system optimal load flow. IEEE, Vol 7,No 2, May.

Murto, P. (1998) Neural Networks Models for Short-term load forecasting. Department of Engineering, Physics and Mathematics. Helsinki University of Technology.

Nahmias S. (2007) "Análisis de la producción y las operaciones", McGrawHill INTERAMERICANA EDITORES, p.p, 57.

Piras, A. Germond, A. Buchenel, B. Imhof K, Jaccard Y, (1996) Heterogeneous Artificial Neural Network for short term electrical load for e casting. IEEE, Vol 11, No 1. Feb.

Shoults, RR. Subbarayan Liu, Manry, MT (1996) Comparison of very short term load forecasting techniques. IEEE, Vol. 11, No 2, May.

Sforma, M. Lamedica, R. Prudenzi, A, Caciotta M. (1996) A neural network based technique for short term forecasting of anomalous load periods. IEEE, Vol 11, No 4. 